\title{
Prognostic Factors and Nomogram Predicting Survival in Diffuse Astrocytoma
}

\author{
Thara Tunthanathip ${ }^{1}$ Sanguansin Ratanalert ${ }^{2}$ Sakchai Sae-heng ${ }^{1} \quad$ Thakul Oearsakul $^{1}$ \\ Ittichai Sakaruncchai ${ }^{1}$ Anukoon Kaewborisutsakul ${ }^{1}$ Thirachit Chotsampancharoen ${ }^{3}$ \\ Utcharee Intusoma ${ }^{4}$ Amnat Kitkhuandee ${ }^{5}$ Tanat Vaniyapong ${ }^{6}$
}

\footnotetext{
${ }^{1}$ Division of Neurosurgery, Department of Surgery, Faculty of Medicine, Prince of Songkla University, Songkhla, Thailand

${ }^{2}$ School of Medicine, Mae Fah Luang University, Chiang Rai, Thailand

${ }^{3}$ Division of Hematology/Oncology, Department of Pediatrics, Faculty of Medicine, Prince of Songkla University, Songkhla, Thailand

${ }^{4}$ Division of Pediatric Neurology, Department of Pediatrics, Faculty of Medicine, Prince of Songkla University, Songkhla, Thailand

${ }^{5}$ Division of Neurosurgery, Department of Surgery, Faculty of Medicine, Khon Kaen University, Khon Kaen, Thailand

${ }^{6}$ Division of Neurosurgery, Department of Surgery, Faculty of

Medicine, Chiang Mai University, Chiang Mai, Thailand
}

\author{
Address for correspondence Thara Tunthanathip, Division of \\ Neurosurgery, Department of Surgery, Faculty of Medicine Prince of \\ Songkla University, Hat Yai, Songkhla, Thailand 90110 \\ (e-mail: tsus4@hotmail.com).
}

\begin{abstract}
Background Prognosis of low-grade glioma are currently determined by genetic markers that are limited in some countries. This study aimed to use clinical parameters to develop a nomogram to predict survival of patients with diffuse astrocytoma (DA) which is the most common type of low-grade glioma.

Materials and Methods Retrospective data of adult patients with DA from three university hospitals in Thailand were analyzed. Collected data included clinical characteristics, neuroimaging findings, treatment, and outcomes. Cox's regression analyses were performed to determine associated factors. Significant associated factors from the Cox regression model were subsequently used to develop a nomogram for survival prediction. Performance of the nomogram was then tested for its accuracy.

Results There were 64 patients with DA with a median age of 39.5 (interquartile range $[I Q R]=20.2$ ) years. Mean follow-up time of patients was 42 months (standard deviation $[S D]=34.3$ ). After adjusted for three significant factors associated with survival were age $\geq 60$ years (hazard ratio $[\mathrm{HR}]=5.8 ; 95 \%$ confidence interval $[\mathrm{Cl}]: 2.09-15.91$ ), motor response score of Glasgow coma scale $<6(\mathrm{HR}=75.5 ; 95 \% \mathrm{Cl}: 4.15-1,369.4)$, and biopsy ( $\mathrm{HR}=0.45 ; 95 \% \mathrm{Cl}$ : 0.21-0.92). To predict 1-year mortality, sensitivity, specific-

Keywords

- diffuse astrocytoma

- nomogram

- survival analysis ity, positive predictive value, negative predictive value, accuracy, and area under the curve our nomogram was $1.0,0.50,0.45,1.0,0.64$, and 0.75 , respectively.

Conclusions This study provided a nomogram predicting prognosis of DA. The nomogram showed an acceptable performance for predicting 1-year mortality.
\end{abstract}

\section{Introduction}

Diffuse astrocytoma (DA) is one type of low-grade glioma (LGG) and classified as WHO (World Health Organization) grade II. DA is a relatively slow-growing brain tumor with a median survival time of 3.9 to 10.8 years. ${ }^{1-6}$ However, DA infiltrates brain parenchyma, making it hard for total resection. The rate of gross-total resection is only 14 to $17 \%^{2,3}$ Therefore, residual tumors become the burden of patients with DA in the long-term follow-up.
DOI https://doi.org/ $10.1055 / \mathrm{s}-0039-3403446$ ISSN 0976-3147.
License terms

(ㅇ)(1) $\Theta \circledast$ 
Low-grade glioma (LGG) comprised of several types of tumor, that is, astrocytoma, oligodendroglioma, and mixed oligoastrocytoma. Associated factors for poor outcomes in patients with LGG are older age, Karnofsky's performance status $(\mathrm{KPS})<70$, prior neurological deficits, tumor size $>6 \mathrm{~cm}$, tumor crossing midline, and nonhemispheric/noncerebellar tumor. ${ }^{6-8}$ Postoperative radiotherapy (RT) is yet a debatable predicting factor for prognosis of LGG. In a study, postoperative conventional RT tends to shorten time to progression and prolonged progression-free survival but not the overall survival. ${ }^{9}$ Another study shows no significant difference in survival between pediatric patients with LGG who received postoperative RT and those who did not. ${ }^{8}$ Nonetheless, there has been limited data about prognosis among subpopulation of patients with LGG including patients with DA.

Nomogram has been used to predict clinical outcome in various groups of diseases, such as malignancies, ${ }^{10,11}$ degenerative diseases, ${ }^{12}$ and metabolic diseases. ${ }^{13}$ From the literature review, there is the heterogeneity of histology in the group of LGG and lack of evidence of the prognostic factors that focuses on diffuse astrocytoma. We aimed to identify prognostic factors associated with survival of patients with diffuse astrocytoma. This study developed the nomogram to predict survival of DA hoping to use in the clinical practice.

\section{Subjects and Methods}

\section{Study Designs and Population}

We reviewed data from three university hospitals in Thailand (Central Nervous System Tumor Registry). Patients who were newly diagnosed with DA within the study period (January 2009 and December 2017) were included. DA was histologically-confirmed by certified pathologists using World Health Organization Classification. ${ }^{14}$ Data collected included demographics, neuroimaging, treatment, and outcome.

The Karnofsky performance status (KPS) score is an assessment tool for functional impairment. Scores run from 100 to 0 (the lowest KPS score has the worst survival probability). KPS scores were also dichotomized into two groups, according to the ability to carry on normal activities (KPS $\geq 80$ ). ${ }^{15}$

Magnetic resonance images (MRI) of the brain were reviewed prospectively by trained neurosurgeons to demonstrate tumor location, tumor size, and other characteristics of the tumor. The postoperative residual tumor was quantified from postoperative MRI or contrast-enhanced computerized tomography (CT) of the brain. The hypervascularization of the tumor defined as the visualizing vascular structures inside a tumor (flow void sign) was reviewed. ${ }^{16}$

The extent of resection was defined using the same definition by Vecht et al. ${ }^{17}$ Gross-total resection was defined as residual tumor seen in postoperative neuroimaging less than $5 \%$. Subtotal resection was defined as residual tumor 5 to $25 \%$ seen in postoperative neuroimaging. Partial resection was defined as visible residual tumor more than $25 \%$. Biopsy was defined as an operation for tissue diagnosis only, and no attempt was made to remove the tumor.

The follow-up data were collected until December 2018. Survival duration was duration from the time of surgery to death or censor (still survived) in December 2018. Follow-up data were collected mainly when patients visited outpatient clinics. Patients (or caregivers) who did not come to the hospital were interviewed by phone. We also checked death records from the local municipality. The study got clearance from the institutional review board of each collaborator (REC number 61-203-10-1).

\section{Nomogram Development and Performance Test}

From the multivariable analysis, the prediction model was constructed from the significant parameters that affect the mortality. A nomogram was developed using the significant parameters $(p<0.05)$ of the method by Zhang et al. ${ }^{18}$ The bootstrap method with 1,000 replicates was used for the internal validity of the model. The validated function in the "rms" package was used to analyze the bias-corrected c-index that evaluated the predictive discrimination of the model. ${ }^{19}$ The concordance index is the probability of concordance between predicted probability and response.

For the individual prediction, the performances of nomogram were evaluated as the binary classifiers (death or living) instead of the death-probability prediction by the self-consistency validation. ${ }^{20}$ The nomogram's sensitivity, specificity, positive predictive value (PPV), negative predictive value (NPV), and accuracy were determined for death in various cut-off point of the total scores. Therefore, the highest performance nomogram with optimal cut-off points was chosen by the receiver operating characteristic (ROC) curve and the area under the receiver operating characteristic (AUC) were plotted. Additionally, AUCs were determined that values $\geq 0.9$ are "excellent," $\geq 0.80$ "good," $\geq 0.70$ "fair," and $<0.70$ "poor." 21

\section{Statistical Analysis}

Clinical characteristics and therapeutic factors were first described using descriptive statistics. Survival curve was constructed using the Kaplan-Meier method. The Cox proportional hazard regression model was used to identify the univariate and multivariate predictors of survival. A $p$-value less than 0.05 was considered statistically significant. The statistical analysis was performed using the $\mathrm{R}$ version 3.4.0 software ( $R$ Foundation, Vienna, Austria). In details, the "rms" package was used for establishing nomogram. ${ }^{19}$ Moreover, ROC and AUC were created by "PlotROC" package. ${ }^{22}$

\section{Results}

The clinical manifestations of the 64 patients with DA are shown in - Table 1. More than half of the DA was slightly dominant in males. The mean age was 39.4 years $(S D=17.3$ ). The patients usually presented with a seizure and a progressive headache. Additionally, $96.9 \%$ of patients had the Glasgow coma scale (GCS) score 15, while 3.1\% had a GCS score of less than 15 . The common tumor location involved the frontal and temporal lobe in 37.5 and $23.4 \%$, respectively. The thalamic tumor was found in $1.6 \%$ of cases. Moreover, the mean tumor volume was $5.6(+\mathrm{SD}=1.8)$ and $92.2 \%$ of the DA was solitary tumors while $7.8 \%$ was multiple tumors. 
Table 1 Clinical characteristics of patients $(n=64)$

\begin{tabular}{|c|c|}
\hline Factor & $n(\%)$ \\
\hline \multicolumn{2}{|l|}{ Age (y) } \\
\hline$<60$ & $55(85.9)$ \\
\hline$\geq 60$ & $9(14.1)$ \\
\hline Median of age (y)(IQR) & $39.5(20.2)$ \\
\hline \multicolumn{2}{|l|}{ Gender } \\
\hline Male & $34(53.1)$ \\
\hline Female & 30 (46.9) \\
\hline Seizure & $34(53.1)$ \\
\hline Progressive headache & $21(32.8)$ \\
\hline Weakness & $16(25.0)$ \\
\hline Visual disturbance & $3(4.7)$ \\
\hline Alteration of consciousness & $2(3.1)$ \\
\hline Ataxic gait & $2(3.1)$ \\
\hline \multicolumn{2}{|c|}{ Preoperative Karnofsky’s performance status } \\
\hline$<80$ & $22(34.4)$ \\
\hline$\geq 80$ & $42(65.6)$ \\
\hline \multicolumn{2}{|l|}{ Major location of the tumor } \\
\hline Frontal & $24(37.5)$ \\
\hline Temporal & $15(23.4)$ \\
\hline Corpus callosum & $7(10.9)$ \\
\hline Parietal & $5(7.8)$ \\
\hline Brainstem & $3(4.7)$ \\
\hline Pineal & $2(3.1)$ \\
\hline Spinal cord & $2(3.1)$ \\
\hline Occipital & $1(1.6)$ \\
\hline Periventricular & $1(1.6)$ \\
\hline Basal ganglion & $1(1.6)$ \\
\hline Thalamus & $1(1.6)$ \\
\hline Sellar/suprasellar & $1(1.6)$ \\
\hline Cerebellum & $1(1.6)$ \\
\hline \multicolumn{2}{|l|}{ Lateralization of tumor } \\
\hline Left & $24(37.5)$ \\
\hline Right & $27(42.2)$ \\
\hline Bilateral & $1(1.6)$ \\
\hline Midline & $12(18.8)$ \\
\hline \multicolumn{2}{|l|}{ Number of tumors } \\
\hline Single & $59(92.2)$ \\
\hline Multiple & $5(7.8)$ \\
\hline Preoperative hydrocephalus & $15(23.4)$ \\
\hline Positive hypervascular signs & $20(31.3)$ \\
\hline Initial leptomeningeal dissemination & $3(4.7)$ \\
\hline Eloquent area & $26(40.6)$ \\
\hline Mean of diameter $(\mathrm{cm})(\mathrm{SD})$ & $5.6(1.8)$ \\
\hline Mean of midline shift (mm) (SD) & $3.3(3.7)$ \\
\hline
\end{tabular}


Table 1 (continued)

\begin{tabular}{|l|l|}
\hline Factor & $n(\%)$ \\
\hline Type of operation & \\
\hline Total resection & $8(12.5)$ \\
\hline Subtotal resection & $7(10.9)$ \\
\hline Partial resection & $27(42.2)$ \\
\hline Biopsy & $22(34.4)$ \\
\hline Radiotherapy & $53(82.8)$ \\
\hline Postoperative Karnofsky's performance status & \\
\hline$<80$ & $29(45.3)$ \\
\hline$\geq 80$ & $35(54.7)$ \\
\hline
\end{tabular}

Abbreviations: IQR, interquartile range; SD, standard deviation.
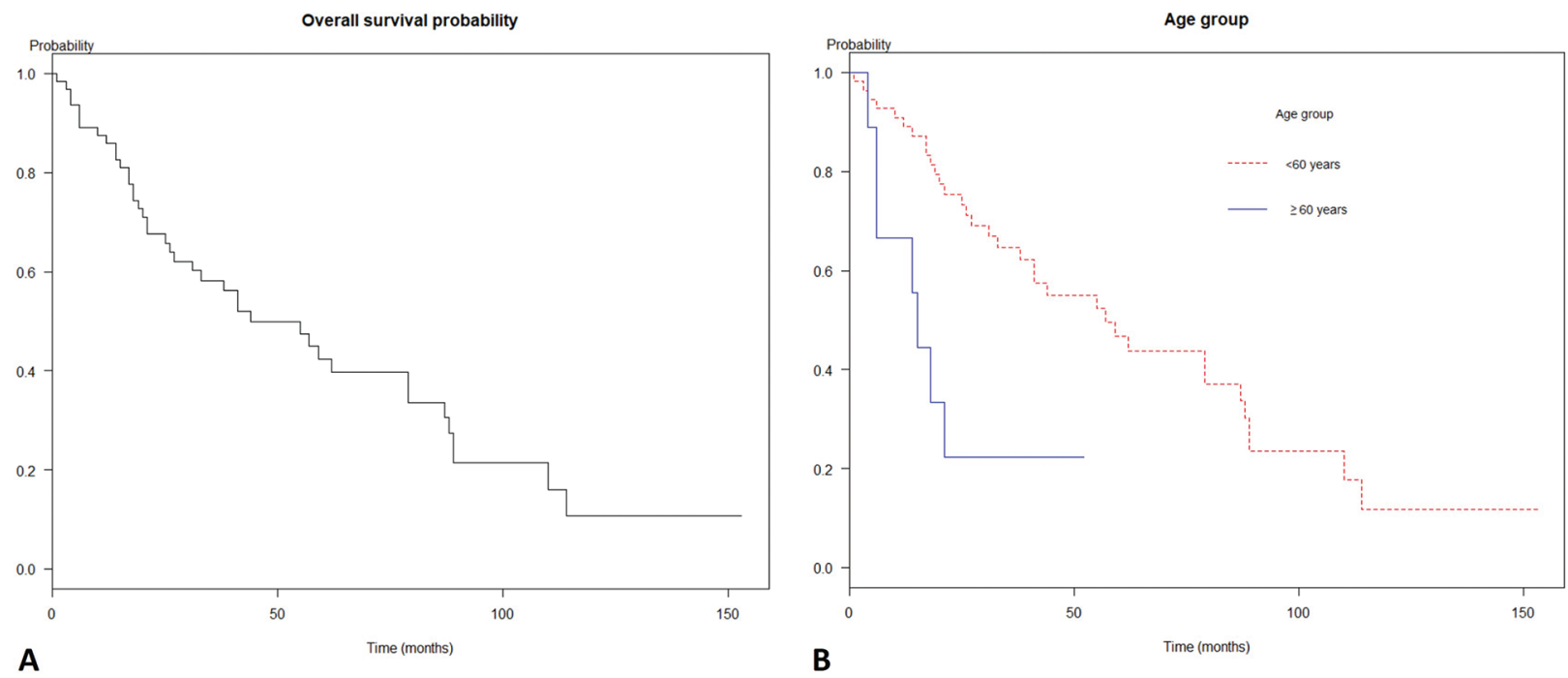

A

Type of surgery
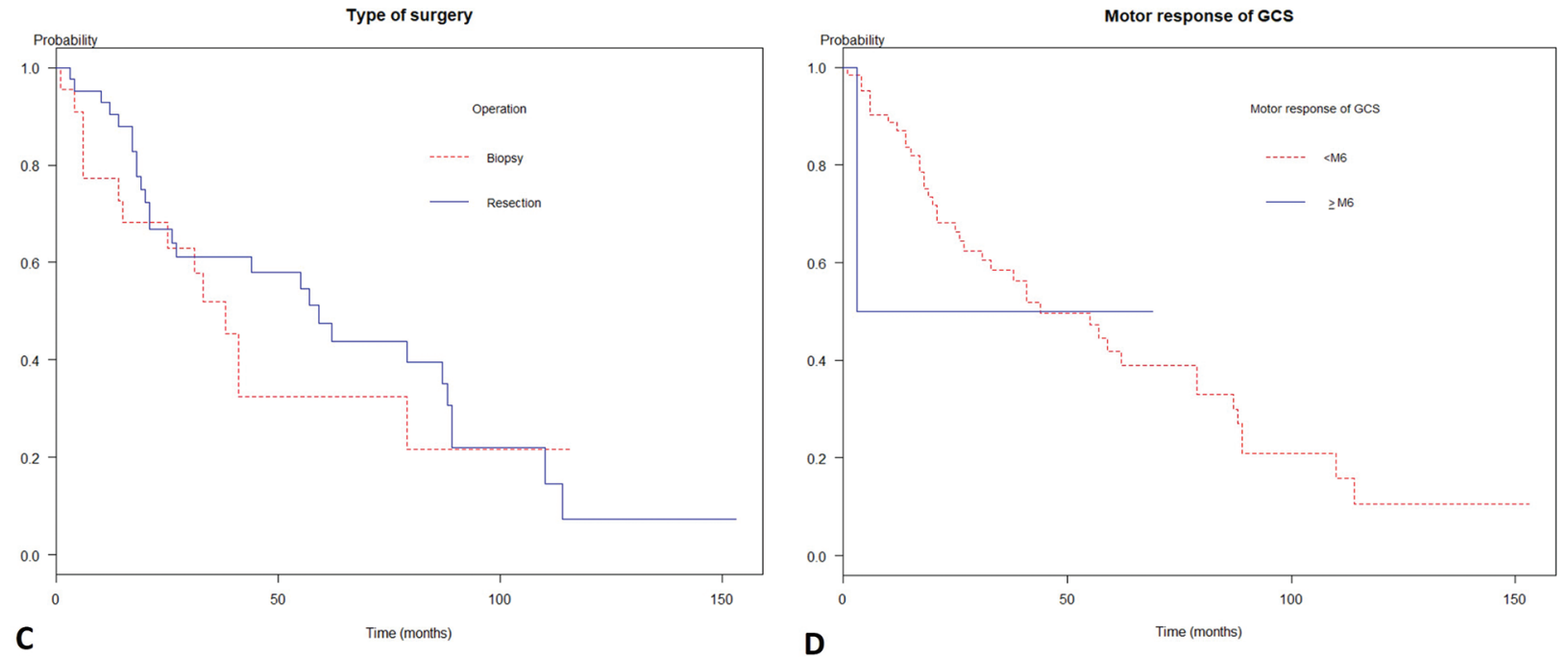

Fig. 1 Survivals of patients with diffuse astrocytoma glioma by predictors. (A) The overall median survival time was 26 months (95\% confidence interval [Cl]: 19.0-41.0). (B) A comparison of survival times among age groups (log-rank test, $p=0.004)$. (C) A comparison of survival times among patients according to motor response groups (log-rank test, $p<0.001$ ). (D) A comparison of survival times among biopsy and resection operations (log-rank test, $p=0.04)$.

In this study, the total resection rate was $12.5 \%$, while the rates of subtotal, partial resection, and biopsy were 10.9, 42.2 , and $34.4 \%$, respectively. Most of the patients (82.8\%) underwent radiotherapy after resection.

\section{Survival Analysis}

The prognosis of the diffuse astrocytoma was pitiable as the Kaplan-Meier curve in - Fig. 1A Mean follow-up time was 42 months ( $S D=34.3$ ). Also, the overall median survival time 
was 44 months (95\% confidence interval [CI]: 31.0-87.0), while the 1-, 2-, and 5-year survival probability were 85.9 , 67.6 , and $42.3 \%$, respectively. Moreover, the malignant transformation was observed in $17.2 \%$ of cases.

According to Cox's proportional hazard regression analysis as shown in - Table 2, the significant parameters for increased death were age 60 years or more group (hazard ratio $[\mathrm{HR}]=4.83, p=0.001$ ), motor response of GCS score less than 6 in groups (HR 39.49, $p=0.009$ ), positive hypervascular sign ( HR $=2.05, p=0.03)$, biopsy $(H R=0.47, p=0.03)$ in univariate analysis. By multivariable analysis, the significant model consisted of age 60 years or more $(H R=5.76$, $p<0.001)$, the motor response score of GCS less than 6 $(\mathrm{HR}=75.47, p=0.003)$, and biopsy $(\mathrm{HR}=0.45, p=0.02)$.

As the Kaplan-Meier curve in - Fig. 1B-D, the median survival time of age less than the 60-year group was 34 months, whereas age 60 or above group was 7 months (log rank test, $p<0.01$ ). The patients with motor response score of GCS less than 6 had median survival time at 40 months while motor response score of GCS equal to 6 had median survival time at a month (log-rank test, $p=0.005$ ). Additionally, the median survival time of biopsy and tumor resection was 22 months and 27 months, respectively (log rank test, $p=0.04$ ).

\section{Nomogram Development and Performance Test}

The nomogram was developed using the significant parameters in the multivariable analysis as shown in - Fig. 2. Additionally, the calibrate plot revealed that the model was nearby the ideal and had a bias-corrected concordance-index value of 0.933 . The nomogram is simple in general practice. For example, a 58-year-old patient (no point) had six scores in the motor response of GCS (no point), and the MRI of the brain showed a left frontal diffuse astrocytoma with positive of the hypervascular sign, and he underwent to total tumor resection (no point) as $\boldsymbol{-}$ Fig. 3 . Therefore, total points equal to 0 points which approximately corresponds to more than $90 \%$ of 1 -year survival probability, more than $70 \%$ of 2 -year survival probability, 35 to $40 \%$ of 5 -year survival probability, and 80 to 90 months of predicted-survival time.

For validation, the performances of nomogram were evaluated as the binary classifiers instead of survival probability. Therefore, nomogram needed to find the optimal cut-off point for highest performance. At an optimal cut-off point of 18 , the nomogram was validated by self-consistency test that sensitivity, specificity, PPV, NPV, accuracy, and AUC were $0.77,0.61,0.25,0.94,0.70$, and 0.70 , respectively for predicting 1 -year mortality as - Fig. 4. Nevertheless, nomogram performance gradually dropped in 2-year and 5-year prediction as - Table 3.

\section{Discussion}

Overall median survival time have been reported between 3.9 and 10.8 years in the LGG which composed of astrocytomas, oligodendrogliomas, and mixed oligoastrocytomas. ${ }^{1-6}$ Spych et al reported LGG had the median survival times of 26.9 months (range: 25.4-46.8 months), whereas the median survival times was 48 months (range: 30 months-138 months) according to the study by Kumthekar et al. ${ }^{2,3}$ Moreover, The 1-year, 3-year, and 5 -year overall survivals of LGG were 88.3, 55.1, and 43.3\%, respectively. ${ }^{2}$ As the present results, the prognosis of our cohort was poorer than prior studies that the median survival time of the cohort was 26 months and the 5-year probability was $22.0 \%$. Since the heterogeneity of the LGG population limits to comparison with the present cohort.

For negative prognostic factors, Pignatti et al reported that age $>40$ years, whereas Okamoto et al reported age $>50$ years associated with the poor prognosis. ${ }^{6}$ Similarly, the present study found the elderly patients had a poor prognosis. Furthermore, the presence of neurologic deficit before surgery was one of the significant prognostic factors, while motor response score of GCS was less than 6 , as well as the poor performance status of the patients was associated with worse outcome in the present study.

Several studied reported that tumor resection significantly impacts the survival of LGGs. ${ }^{23-25}$ The result in the present study concordances the prior studies that biopsy was significantly associated with increased mortality. Furthermore, the effect of RT is controversial for improving outcome and prognosis. LGGs treated with postoperative RT which was highly correlated with overall survival ${ }^{3,9}$ in prior studies, whereas other studies reported that postoperative RT was no statistically significant difference in survival was seen between the postoperative RT and non-RT groups., ${ }^{2,8,26}$ However, the heterogeneity of the study population of LGGs was observed in previous studies. In the present cohort of DA, the radiotherapy following surgery was no statistically significant association with overall survival rate. From the literature review, a few studies focused on the prognosis of the DA group since the limitation of sample size were considered. Nevertheless, the present study, which focused on the specific DA group, had the strength in the homogeneity of the study population. To the best of our knowledge, this is the first study mentioned to evaluate and validate nomogram which is specific to DA.

Gorlia et al proposed nomogram for predicting survival of patients with LGGs including astrocytoma, oligodendroglioma, and mixed oligoastrocytoma. The final overall survival model, independent prognostic factors were identified as time since first LGG symptoms (HR = 0.67, $p=0.009)$, Medical Research Council (MRC) neurological score (HR $=1.51$, $p=0.0001)$, independent confirmation of astrocytoma ( HR $=1.96, p<0.001)$, and tumor size $(\mathrm{HR}=1.74, p=0.001)$. However, there is a limitation of validation because the prediction of nomogram is probability at each time point and survival time. ${ }^{27}$ For example, predicted the 1 -year probability of nomogram is $50 \%$ what we should interpret these results for an individual in the real-world applications. Therefore, we proposed nomogram validation as binary classifiers each time point with the optimal cut-off point in the present study. The nomogram of our cohort had acceptable performances for predicting 1-year mortality that had a high level of sensitivity and accuracy. For general practice, the nomogram could be applied as the screening tool for advising patients and managing treatment strategies. However, nomogram's 
Table 2 Factors associated with the death of patients with diffuse astrocytoma

\begin{tabular}{|c|c|c|c|c|}
\hline & Univariate analysis & & Multivariable analysis & \\
\hline Factor & Odds ratio $(95 \% \mathrm{Cl})$ & $p$-Value & Odds ratio $(95 \% \mathrm{CI})$ & $p$-Value \\
\hline \multicolumn{5}{|l|}{ Gender } \\
\hline Male & Ref. & & & \\
\hline Female & $1.16(0.61-2.20)$ & 0.63 & & \\
\hline \multicolumn{5}{|l|}{ Age (y) } \\
\hline$<60$ & Ref. & & & \\
\hline$\geq 60$ & $4.83(1.86-12.55)$ & 0.001 & $5.76(2.09-15.91)$ & $<0.001$ \\
\hline Aphasiaa & $4.50(0.56-36.0)$ & 0.15 & & \\
\hline Seizure $^{a}$ & $1.08(0.56-2.08)$ & 0.80 & & \\
\hline \multicolumn{5}{|l|}{ Motor response of GCS } \\
\hline Equal 6 & Ref. & & Ref. & \\
\hline Less than 6 & $39.49(2.47-631.49)$ & 0.009 & $75.47(4.15-1369.4)$ & 0.003 \\
\hline \multicolumn{5}{|l|}{$\begin{array}{l}\text { Preoperative Karnofsky performance } \\
\text { status }\end{array}$} \\
\hline$<80$ & Ref. & & & \\
\hline$\geq 80$ & $1.29(0.67-2.51)$ & 0.43 & & \\
\hline \multicolumn{5}{|l|}{ Location } \\
\hline Frontal lobe ${ }^{a}$ & $1.06(0.55-2.05)$ & 0.84 & & \\
\hline Temporal lobe & $0.83(0.49-1.68)$ & 0.69 & & \\
\hline Parietal lobe & $0.67(0.20-2.28)$ & 0.52 & & \\
\hline Brainstem $^{\mathrm{a}}$ & $1.17(0.15-8.71)$ & 0.87 & & \\
\hline Thalamus/basal gangliona & $2.85(0.65-12.32)$ & 0.16 & & \\
\hline Corpus callosuma & $2.20(0.63-7.71)$ & 0.21 & & \\
\hline Sellar/suprasellar region ${ }^{\mathrm{a}}$ & $0.20(0.02-1.75)$ & 0.14 & & \\
\hline Spinal cord ${ }^{\mathrm{a}}$ & $2.59(0.32-20.52)$ & 0.36 & & \\
\hline \multicolumn{5}{|l|}{ Lateralization of tumor } \\
\hline Left & Ref. & & & \\
\hline Right & $1.45(0.73-2.88)$ & 0.28 & & \\
\hline Midline & $1.36(0.53-3.48)$ & 0.51 & & \\
\hline Eloquent area ${ }^{\mathrm{a}, \mathrm{b}}$ & $0.87(0.46-1.65)$ & 0.68 & & \\
\hline \multicolumn{5}{|l|}{ Number of tumors } \\
\hline Single & Ref. & & & \\
\hline Multiple & $2.18(0.75-6.28)$ & 0.14 & & \\
\hline Positive hypervascular signa & $2.08(1.04-4.12)$ & 0.03 & $1.81(0.88-3.75)$ & 0.10 \\
\hline Leptomeningeal dissemination ${ }^{a}$ & $1.59(0.37-6.73)$ & 0.52 & & \\
\hline Preoperative hydrocephalus ${ }^{\mathrm{a}}$ & $1.09(0.51-2.30)$ & 0.81 & & \\
\hline \multicolumn{5}{|l|}{ Midline shift } \\
\hline$<0.5$ & Ref. & & & \\
\hline$\geq 0.5$ & $1.20(0.59-2.45)$ & 0.59 & & \\
\hline \multicolumn{5}{|l|}{ Maximum diameter $(\mathrm{cm})$} \\
\hline$<3$ & Ref. & & & \\
\hline
\end{tabular}


Table 2 (continued)

\begin{tabular}{|c|c|c|c|c|}
\hline & Univariate analysis & & Multivariable analysis & \\
\hline Factor & Odds ratio $(95 \% \mathrm{Cl})$ & $p$-Value & Odds ratio $(95 \% \mathrm{Cl})$ & $p$-Value \\
\hline$\geq 3$ & $0.73(0.30-1.78)$ & 0.49 & & \\
\hline \multicolumn{5}{|c|}{ Type of operation } \\
\hline Biopsy & Ref. & & Ref. & \\
\hline Resection & $0.47(0.23-0.95)$ & 0.03 & $0.45(0.21-0.92)$ & 0.02 \\
\hline \multicolumn{5}{|c|}{$\begin{array}{l}\text { Postoperative Karnofsky’s perfor- } \\
\text { mance status }\end{array}$} \\
\hline$<80$ & Ref. & & & \\
\hline$\geq 80$ & $0.98(0.52-1.85)$ & 0.95 & & \\
\hline \multicolumn{5}{|l|}{ Radiotherapy } \\
\hline No & Ref. & & & \\
\hline Yes & $0.86(0.35-2.06)$ & 0.73 & & \\
\hline
\end{tabular}

Abbreviations: $\mathrm{Cl}$, confidence interval; Ref., reference.

aData show only "yes group” while reference groups (no group) are hidden.

bEloquent area defined tumor involved motor cortex, sensory cortex, visual center, speech center, basal ganglion, hypothalamus, thalamus, brainstem, dentate nucleus.

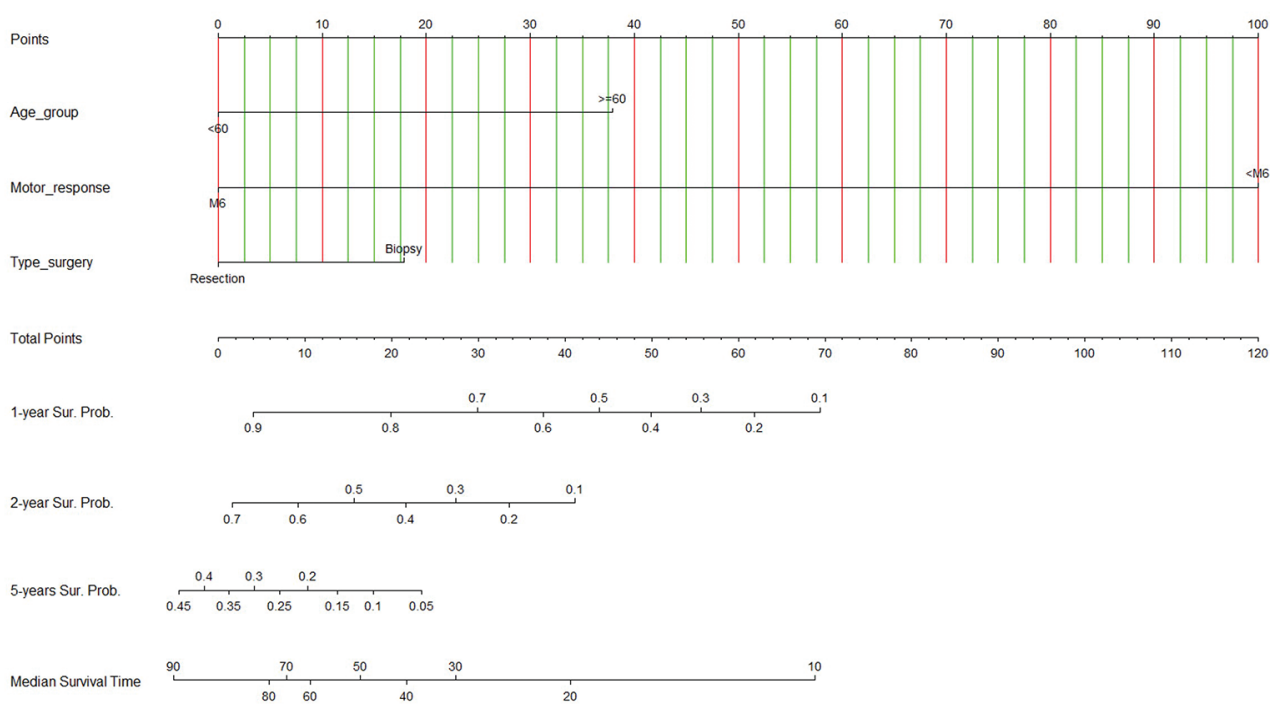

Fig. 2 Nomogram predicting 1-year, 2-year, 5-year survival probabilities (Sur. Probs.) and median survival time (months). To use the nomogram, draw a straight line upward from the patient's characteristics of age group, motor response, type of surgery to the upper points scale, the sums of the scores of all variables. Then, draw another straight line down from the scale of the total points through the 1-year, 2-year, 5 -year, and median survival times. This is the probability of the presence of prognosis in an individual.

performances dropped for predicting 2- and 5-year mortality that needs external validation in the future.

\section{Limitations}

Certain limitations of the present study should be acknowledged. The IDH1 mutation of DAs did not perform in the present study because these genetic investigations have not routinely estimated in Thailand. Moreover, the possibility of bias and confounding factors cannot be excluded from the retrospective study. However, we presented to adjust the model by multivariable analysis for controlling this limitation ${ }^{28}$

\section{Conclusion}

We provided nomogram predicting prognosis of a patient with DA. The nomogram was acceptable performance for predicting 1-year mortality. The tool is a good clinical utility for optimizing therapeutic approaches and counseling patients. 

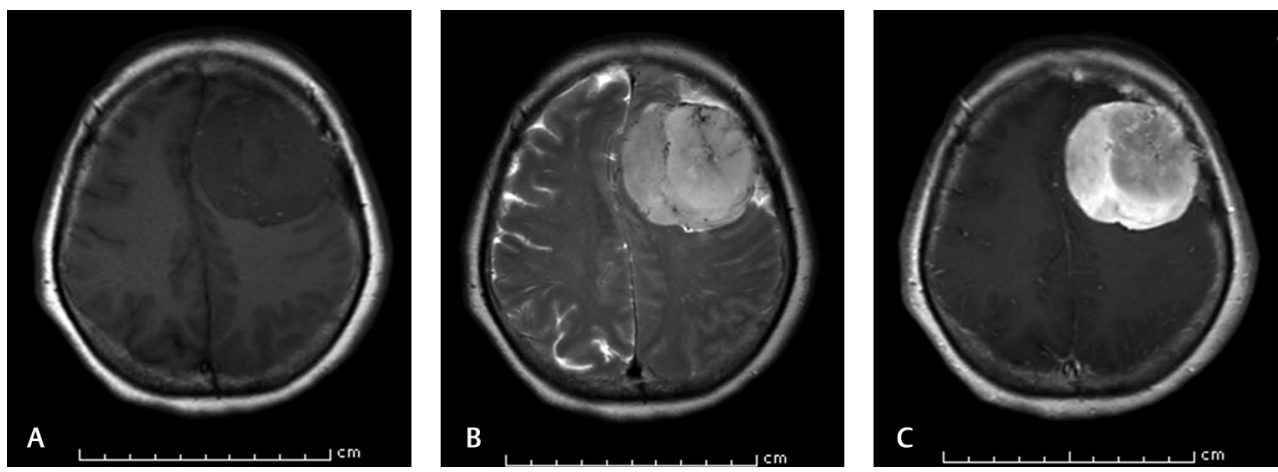

Fig. 3 Brain MRI of a 58-year-old patient who survived at 4-year-follow-up. (A) Axial T1-weighted image shows left hypointense frontal mass. (B). Axial T2-weighted image demonstrates the intratumoral flow void sign. (C). Axial T1-weighted image shows vivid enhancement after contrast injection. MRI, magnetic resonance imaging.
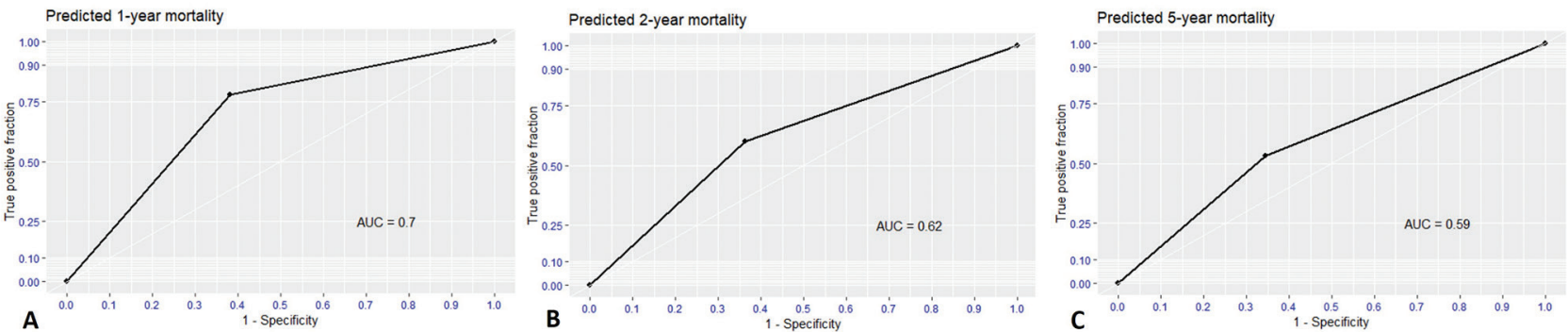

Fig. 4 Receiver operating characteristic (ROC) curve and area under the curve (AUC) of predicted mortality as binary classifiers using cut-offpoint of 18. (A) 1-year predicted mortality, (B) 2-year predicted mortality, and (C) 5-year predicted mortality.

Table 3 Performance test of a nomogram predicting 1-, 2-, and 5-year mortality of diffuse astrocytoma at cut-off 18 points

\begin{tabular}{|l|l|l|l|l|l|}
\hline Prediction & Sensitivity & Specificity & PPV & NPV & Accuracy \\
\hline 1-year mortality & 0.77 & 0.61 & 0.25 & 0.94 & 0.70 \\
\hline 2-year mortality & 0.60 & 0.63 & 0.42 & 0.77 & 0.62 \\
\hline 5-year mortality & 0.53 & 0.65 & 0.60 & 0.58 & 0.59 \\
\hline
\end{tabular}

Abbreviations: NPV, negative predictive value; PPV, positive predictive value.

\section{Key Messages}

To the best of authors' knowledge, this article is the first one to mention the nomogram predicting prognosis of diffuse astrocytoma. Moreover, we proposed that nomogram can be used in the binary outcome for simplifying nomogram application in the clinical practice.

\section{Authors' Contributions}

T.T., S.R., U.I., A.K., and T.V. participated in study design and data collection plan. T.T., S.S., T.O., I.S., A.K., S.R., A.K., and T.V. supervised data collection. T.T., S.R., A.K., and T.V. contributed in data extraction, data management, and quality control. T.T. analyzed the data and drafted the manuscript. All authors contributed substantially to manuscript revision. T.T. takes responsibility for this paper as a whole.

\section{Funding}

This study was funded by the faculty of Medicine at Prince of Songkla University, Thailand.

\section{Conflict of Interest}

None declared.

\section{Acknowledgment}

The authors would like to offer special thanks to Associate Prof. Paramee Thongsuksai for advice about the manuscript preparation. Additionally, the authors would like to thank Mrs. Supaporn Sainamsai for interdepartment and interinstitutes coordination.

\section{References}

1 Daniels TB, Brown PD, Felten SJ, et al. Validation of EORTC prognostic factors for adults with low-grade glioma: a report using intergroup 86-72-51. Int J Radiat Oncol Biol Phys 2011;81(1):218-224

2 Kumthekar P, Patel V, Bridge C, et al. Prognosis of older patients with low-grade glioma: a retrospective study. Integr Cancer Sci Ther 2017;4(5):1-5

3 Spych M, Gottwald L, Jesień-Lewandowicz E, Sztajer S, Fijuth J. Response to postoperative radiotherapy as a prognostic factor for patients with low-grade gliomas. Oncol Lett 2012;4(3):455-460

4 Bourne TD, Schiff D. Update on molecular findings, management and outcome in low-grade gliomas. Nat Rev Neurol 2010;6(12):695-701 
5 Ohgaki $\mathrm{H}$, Kleihues P. Population-based studies on incidence, survival rates, and genetic alterations in astrocytic and oligodendroglial gliomas. J Neuropathol Exp Neurol 2005;64(6):479-489

6 Okamoto Y, Di Patre PL, Burkhard C, et al. Population-based study on incidence, survival rates, and genetic alterations of low-grade diffuse astrocytomas and oligodendrogliomas. Acta Neuropathol 2004;108(1):49-56

7 Pignatti F, van den Bent M, Curran D, et al. European Organization for Research and Treatment of Cancer Brain Tumor Cooperative GroupEuropean Organization for Research and Treatment of Cancer Radiotherapy Cooperative Group. Prognostic factors for survival in adult patients with cerebral low-grade glioma. J Clin Oncol 2002;20(8):2076-2084

8 Fisher BJ, Leighton CC, Vujovic O, Macdonald DR, Stitt L. Results of a policy of surveillance alone after surgical management of pediatric low grade gliomas. Int J Radiat Oncol Biol Phys 2001;51(3):704-710

9 Karim AB, Afra D, Cornu P, et al. Randomized trial on the efficacy of radiotherapy for cerebral low-grade glioma in the adult: European Organization for Research and Treatment of Cancer Study 22845 with the Medical Research Council study BRO4: an interim analysis. Int J Radiat Oncol Biol Phys 2002;52(2):316-324

10 Graefen M, Karakiewicz PI, Cagiannos I, et al. A validation of two preoperative nomograms predicting recurrence following radical prostatectomy in a cohort of European men. Urol Oncol 2002;7(4):141-146

11 Liang W, Zhang L, Jiang G, et al. Development and validation of a nomogram for predicting survival in patients with resected non-small-cell lung cancer. J Clin Oncol 2015;33(8):861-869

12 Devin CJ, Bydon M, Alvi MA, et al. A predictive model and nomogram for predicting return to work at 3 months after cervical spine surgery: an analysis from the Quality Outcomes Database. Neurosurg Focus 2018;45(5):E9

13 Dogan HS, Altan M, Citamak B, Bozaci AC, Karabulut E, Tekgul S. A new nomogram for prediction of outcome of pediatric shock-wave lithotripsy. J Pediatr Urol 2015;11(2):84.e1-84.e6

14 Komori T. The 2016 WHO classification of tumours of the central nervous system: the major points of revision. Neurol Med Chir (Tokyo) 2017;57(7):301-311

15 Karnofsky DA, Abelmann WH, Craver LF, Burchenal JH. The use of the nitrogen mustards in the palliative treatment of carcinoma - with particular reference to bronchogenic carcinoma. Cancer 1948;1:634-656
16 Tunthanathip T, Ratanalert S, Sae-Heng S, Oearsakul T. Butterfly tumor of the corpus callosum: clinical characteristics, diagnosis, and survival analysis. J Neurosci Rural Pract 2017;8(Suppl 1):S57-S65

17 Vecht CJ, Avezaat CJ, van Putten WL, Eijkenboom WM, Stefanko SZ. The influence of the extent of surgery on the neurological function and survival in malignant glioma. A retrospective analysis in 243 patients. J Neurol Neurosurg Psychiatry 1990;53(6):466-471

18 Zhang Z, Kattan MW. Drawing nomograms with R: applications to categorical outcome and survival data. Ann Trans Med 2017;5(10):211

19 Harrell FE Jr. Package 'rms'. 2019. Available at: https://cran. r-project.org/web/packages/rms/rms.pdf. Accessed January 29, 2019

20 Gergen KJ, Morse SJ. Self-consistency: measurement and validation. Proc Ann Conv Am Psych Assoc 1967;2:207-208

21 Swets JA. ROC analysis applied to the evaluation of medical imaging techniques. Invest Radiol 1979;14(2):109-121

22 Sachs MC, Corty RW. Package 'plotROC' 2019. Available at: https://cran.r-project.org/web/packages/plotROC/plotROC. pdf. Accessed January 29, 2019

23 Patel T, Bander ED, Venn RA, et al. The role of extent of resection in IDH1 wild-type or mutant low-grade gliomas. Neurosurgery 2018;82(6):808-814 10.1093/neuros/nyx265

24 A Mirza F, Shamim MS. Extent of resection and timing of surgery in adult low grade glioma. J Pak Med Assoc 2017;67(6):959-961

25 Nitta M, Muragaki Y, Maruyama T, et al. Updated therapeutic strategy for adult low-grade glioma stratified by resection and tumor subtype. Neurol Med Chir (Tokyo) 2013;53(7):447-454

26 van den Bent MJ, Afra D, de Witte O, et al; EORTC Radiotherapy and Brain Tumor Groups and the UK Medical Research Council. Long-term efficacy of early versus delayed radiotherapy for low-grade astrocytoma and oligodendroglioma in adults: the EORTC 22845 randomised trial. Lancet 2005;366(9490):985-990

27 Gorlia T, Wu W, Wang M, et al. New validated prognostic models and prognostic calculators in patients with low-grade gliomas diagnosed by central pathology review: a pooled analysis of EORTC/RTOG/NCCTG phase III clinical trials. Neurooncol 2013;15(11):1568-1579

28 Mann CJ. Observational research methods. Research design II: cohort, cross sectional, and case-control studies. Emerg Med J 2003;20(1):54-60 